\title{
SOME LINEAR TOPOLOGICAL PROPERTIES OF SEPARABLE FUNCTION ALGEBRAS ${ }^{1}$
}

\section{A. PEECZYŃSKI ${ }^{2}$}

1. Introduction. Let $C(S)$ be the Banach algebra of all continuous complex-valued functions on a compact Hausdorff space $S$ with the norm $\|f\|=\sup _{s \in S}|f(s)|$.

By a function algebra we mean a closed subalgebra of $C(S)$ which contains constant functions and separates the points of $S$.

In this paper we study some linear topological properties of separable ( $=S$ metrizable) function algebras. In other words, we are interested in the properties of a Banach space which is either linearly homeomorphic or linearly isometric ${ }^{3}$ to a function algebra on a compact metric space. If $S$ is countable, then $C(S)$ is the unique function algebra on $S$ [16]. Therefore for $S$ metrizable the only interesting case is when $S$ is uncountable.

Our main result (Theorem 1) shows that in this case the function algebras still possess several properties which are possessed by the space $C(S)$. In particular, if $A$ is a function algebra on a compact uncountable metric space $S$, then every separable normed linear space is isometric to a closed linear subspace of $A$.

2. Notation. $M(S), S$ compact Hausdorff will denote the usual Banach space of all complex finite regular Borel measures on $S$, the dual of the space $C(S)$. For $\mu$ in $M(S)$ we shall employ the notation $\mu(f)=\int_{S} f d \mu$. The dual space to a normed linear space $X$ will be denoted by $X^{*}$.

A function $g$ in $C(S)$ peaks on the subset $T \subset S$ provided $g^{-1}(\|g\|)=T$ and $|g(s)|<\|g\|$ for $s \in S \backslash T$. If $T=\{t\}$ is a one-point set, we shall say that $g$ peaks at $t$. If $A$ is a function algebra on a compact metric space $S$, then the set

$$
B(A)=\{s \in S: \text { there is some } g \in A \text { such that } g \text { peaks at } s\}
$$

Presented to the Society, January 24, 1966 under the title Some topological and linear topological properties of separable function algebras; received by the editors July 14, 1966.

1 The results of this paper were announced in the Notices Amer. Math. Soc. 13 (1966), 66.

2 Supported in part by the National Science Foundation through Grant GP-3579.

${ }^{3}$ We shall use the term "linearly homeomorphic" instead of "isomorphic" and "linearly isometric" instead of "isometrically isomorphic." For the definition of these notions, cf. [5, p. 65]. 
is called the Choquet boundary for $A$ (Bishop [1], cf. also [15, p. 53]). The set $B(A)$ is an absolute $G_{\delta}$, i.e., it is a $G_{\delta}$ in a compact metric space $S$.

A (nonempty) subset $S_{0}$ of a topological space $S$ is said to be a (proper) perfect set if $S_{0}$ is closed and if it is dense in itself, i.e., every point of $S_{0}$ is a limit point of $S_{0}$. Finally if $T$ is a subset of a topological space $S$, then int $T$ denotes the interior of $T$; if $S$ is metric, then $d\left(s^{\prime}, s^{\prime \prime}\right)$ denotes the distance between points $s^{\prime}$ and $s^{\prime \prime}$ in $S$.

3. "Peano curves" in function algebras. In this section we shall show that if $S$ is uncountable, then in any function algebra on $S$ there are functions whose range is the whole unit disc

$$
K=\{z:|z| \leqq 1\}
$$

(like the Peano curve which maps the unit interval onto the unit square).

Proposition 1. Let $A$ be a function algebra on a compact metric space $S$ and let $S_{0}$ be a proper perfect compact subset of $B(A)$. Then there is a function $h$ in $A$ such that $h(S)=K$ and $h\left(S \backslash S_{0}\right) \subset$ int $K$.

Proof. Since $S \backslash S_{0}$ is an $F_{\sigma}$, there is an increasing sequence of closed sets $F_{1} \subset F_{2} \subset \cdots$ such that $S \backslash S_{0}=\bigcup_{n=1}^{\infty} F_{n}$. Let us set

$$
K_{n}=\left\{z \in K:|z| \leqq 1-2^{-n}\right\} \quad \text { for } n=1,2, \cdots .
$$

We shall define by induction a sequence $\left(f_{n}\right)$ in $A$ satisfying the following properties.

(1) $\left\|f_{n}\right\|<1$,

(2) there is a finite set $Z_{n}$ in $S_{0}$ such that $f_{n}\left(Z_{n}\right) \subset$ int $K_{n}$ and $f_{n}\left(Z_{n}\right)$ is a $2^{-n}$ net for $K_{n+1}$,

(3) $\left\|f_{n+1}-f_{n}\right\|<2^{-n}$,

(4) if $s \in F_{m}$, then $\left|f_{n}(s)\right|<\frac{1}{2}\left(1+\left\|f_{m}\right\|\right)(1 \leqq m \leqq n ; n=1,2, \cdots)$. Let us set $f_{0}=0$ and suppose that for $0 \leqq j \leqq n$ the functions $f_{j}$ satisfying (1) - (4) have been defined. By (2) there are $s_{1}, s_{2}, \cdots, s_{N}$ in $S_{0}$ such that the set $U_{k=1}^{N}\left\{w_{k}\right\}$ is a $2^{-n}$-net for $K_{n+1}$ where $w_{k}=f_{n}\left(s_{k}\right)$ $\in$ int $K_{n}$ for $k=1,2, \cdots, N$. Let $Z=\bigcup_{j=1}^{M}\left\{z_{j}\right\}$ be a $3 \cdot 2^{-n-3}$-net for $K_{n+2}$ such that $\left|z_{j}\right|<1-9 \cdot 2^{-n-4}$. We enumerate elements of $Z$ in such a way that

$$
\left|w_{k}-z_{j}\right|<2^{-n} \text { for } M(k-1)<j \leqq M(k) \quad(k=1,2, \cdots, N)
$$

where $0=M(0)<M(1)<\cdots<M(N)=M$. Let

$$
\sigma_{1}=\max _{1 \leqq k \leqq N} \max _{M(k-1)<j \leqq M(k)}\left|w_{k}-z_{j}\right| ; \quad \sigma_{2}=\max _{1 \leqq k \leqq n}\left|w_{k}\right|,
$$


(6) $\epsilon=\min \left(2^{-n}-\sigma_{1} ; 1-2^{-n}-\sigma_{2} ; 1-\left\|f_{n}\right\|\right)$.

Then $2^{-n}>\epsilon>0$. Since $f_{n}$ is uniformly continuous on $S$ we can choose $\delta>0$ such that for arbitrary $s^{\prime}$ and $s^{\prime \prime}$ in $S$

(7) if $d\left(s^{\prime}, s^{\prime \prime}\right)<\delta$, then $\left|f_{n}\left(s^{\prime}\right)-f_{n}\left(s^{\prime \prime}\right)\right|<\frac{1}{4} \epsilon$.

Since $S_{0}$ is a perfect set, there are $t_{j}$ in $S_{0}$ such that $d\left(s_{k}, t_{j}\right)<\frac{1}{2} \delta$ for $M(k-1)<j \leqq M(k) ; t_{p} \neq t_{q}$ for $p \neq \neq q$. Thus, since $w_{k}=f_{n}\left(s_{k}\right)$, we get (for $M(k-1)<j \leqq M_{k} ; k=1,2, \cdots, N$ )

(8) $\left|f_{n}\left(t_{j}\right)-z_{j}\right| \leqq\left|w_{k}-z_{j}\right|+\left|f_{n}\left(t_{j}\right)-f_{n}\left(s_{k}\right)\right|<2^{-n}-\frac{3}{4} \epsilon$. Let

$$
\delta_{1}=\frac{1}{3} \min \left(\delta, \min _{p \neq q} d\left(t_{p}, t_{q}\right)\right)
$$

Choose $g_{j}$ in $A$ for $j=1,2, \cdots, M$ such that

(9) $\left\|g_{j}\right\|=\left|g_{j}\left(t_{j}\right)\right| ; g_{j}\left(t_{j}\right)=z_{j}-f_{n}\left(t_{j}\right)$,

(10) if $d\left(s, t_{j}\right)>\delta_{1}$, then $\left|g_{j}(s)\right|<(16 M)^{-1} \epsilon$,

(11) if $s \in F_{m}$, then $\left|g_{j}(s)\right|<M^{-1}\left[\frac{1}{2}\left(1+\left\|f_{m}\right\|\right)-\left|f_{n}(s)\right|\right]$ $(m=1,2, \cdots, n)$.

To construct $g_{j}$ choose arbitrary $h_{j}$ in $A$ such that $1=h_{j}\left(t_{j}\right)>\left|h_{j}(s)\right|$ for $s \in S \backslash\left\{t_{j}\right\}$. Since $t_{j} \in B(A)$, such $h_{j}$ exists $(j=1,2, \cdots, M)$. Then put

$$
g_{j}=\left[z_{j}-f_{n}\left(t_{j}\right)\right] h_{j}^{p(j)}
$$

where the integer $p(j)$ is chosen such that for $j=1,2, \cdots, M$,

if $d\left(s, t_{j}\right)>\delta_{1}$, then $\left|h_{j}(s)\right|^{p(j)}<(16 M)^{-1} \boldsymbol{\epsilon}$;

if $s \in F_{m}$, then $\left|h_{j}(s)\right|^{p(j)}<M^{-1} \inf _{s \in F_{m}}\left[\frac{1}{2}\left(1+\left\|f_{m}\right\|\right)-\left|f_{n}(s)\right|\right]$ $(m=1,2, \cdots, n)$.

Let us set

$$
g=\sum_{j=1}^{M} g_{j} ; \quad f_{n+1}=f_{n}+g .
$$

We shall estimate the norms of $g$ and $f_{n+1}$. Let $s \in S$. Let us consider two cases:

$1^{\circ} . d\left(s, t_{j}\right)>\delta_{1}$ for all $j$. Then, by (6), (10) and (12),

$$
|g(s)| \leqq \sum_{j=1}^{M}\left|g_{j}(s)\right|<\frac{\epsilon}{16}<2^{-n},
$$

and

$$
\left|f_{n+1}(s)\right| \leqq\left|f_{n}(s)\right|+|g(s)| \leqq\left\|f_{n}\right\|+\epsilon / 16<1 .
$$

$2^{\circ}$. There is exactly the one index $j(s)$ such that $d\left(s, t_{j(8)}\right) \leqq \delta_{1}$. Then by (5), (6), (10) and (12) 


$$
\begin{aligned}
|g(s)| & \leqq\left\|g_{j(s)}\right\|+\sum_{j \neq j(s)}\left|g_{j}(s)\right| \leqq\left|z_{j(s)}-f_{n}\left(t_{j(s)}\right)\right|+\frac{\epsilon}{16} \\
& <2^{-n}-\frac{\epsilon}{4} .
\end{aligned}
$$

Let us choose $k(s)$ such that $M(k(s)-1)<j(s) \leqq M(k(s))$. Then, since $d\left(s_{k(s)}, t_{j(s)}\right)<\frac{1}{2} \delta$ and $\delta_{1}<\frac{1}{2} \delta$, we get

$$
d\left(s_{k(s)}, s\right) \leqq d\left(s_{k(s)} t_{j(s)}\right)+d\left(s, t_{j(s)}\right)<\delta .
$$

Thus $\left|f_{n}\left(s_{k(s)}\right)-f_{n}(s)\right|<\frac{1}{4} \epsilon$. Then by (7), (8) and (9),

$$
\begin{aligned}
\left|f_{n+1}(s)\right| \leqq & \left|f_{n}(s)\right|+|g(s)| \leqq\left|f_{n}\left(s_{k(s)}\right)\right|+\left|f_{n}\left(s_{k(s)}-f_{n}(s)\right)\right| \\
& +|g(s)|<\left|w_{k(s)}\right|+2^{-n} .
\end{aligned}
$$

Thus since $w_{k(s)} \in \operatorname{int} K_{n},\left|f_{n+1}(s)\right|<1$.

It follows from the choice of $\delta_{1}$ that, for every $s \in S$, either $1^{\circ}$ or $2^{\circ}$ is satisfied. Thus

$$
\left\|f_{n+1}\right\|<1 \text { and }\left\|f_{n+1}-f_{n}\right\|=\|g\|<2^{-n} .
$$

Now we shall check that the set $\bigcup_{j=1}^{M}\left\{f_{n+1}\left(t_{j}\right)\right\} \subset$ int $K_{n+1}$ is a $2^{-n-1}$ net for $K_{n+2}$. It follows from (9) that

$$
f_{n+1}\left(t_{j}\right)=\left(f_{n}\left(t_{j}\right)+g_{j}\left(t_{j}\right)\right)+\sum_{p \neq j} g_{p}\left(t_{j}\right)=z_{j}+\sum_{p \neq j} g_{p}\left(t_{j}\right) .
$$

Hence

$$
\left|f_{n+1}\left(t_{j}\right)-z_{j}\right|<2^{-n-4} \quad(j=1,2, \cdots, M)
$$

because by (10), $\sum_{p \neq j}\left|g_{p}\left(t_{j}\right)\right|<2^{-4} \epsilon<2^{-n-4}$.

Since $\left|z_{j}\right|<1-9 \cdot 2^{-n-4}$ for $j=1,2, \cdots, M$ and since $U_{j=1}^{M}\left\{z_{j}\right\}$ is a $3 \cdot 2^{-n-3}$-net for $K_{n+2}$, the inequality (13) implies that $\left|f_{n+1}\left(t_{j}\right)\right|<1$ $-2^{-n-1}$ for $j=1,2, \cdots, M$ and $\bigcup_{j=1}^{M}\left\{f_{n+1}\left(t_{j}\right)\right\}$ is a $2^{-n-1}$-net for $K_{n+2}$.

Finally if $s \in F_{m}(m=1,2, \cdots, n)$, then by (11) $\sum_{j=1}^{M}\left|g_{j}(s)\right|$ $<\frac{1}{2}\left(1+\left\|f_{m}\right\|\right)-\left|f_{n}(s)\right|$. Therefore $\left|f_{n+1}(s)\right|<\frac{1}{2}\left(1+\left\|f_{m}\right\|\right)$. Clearly if $s \in F_{n+1}$ then $\left|f_{n+1}(s)\right| \leqq\left\|f_{n+1}\right\|<\frac{1}{2}\left(1+\left\|f_{n+1}\right\|\right)$, because we have already checked that $\left\|f_{n+1}\right\|<1$. This completes the induction.

To complete the proof we define

$$
h=\lim _{n} f_{n} .
$$

It follows immediately from (1)-(4) that $h$ has the desired properties.

Corollary 1. Let $A$ be a function algebra on $S, S$ compact and 
metric. Let $S_{0}$ be a proper perfect compact subset of $B(A)$. Then there is $g$ in $A$ which peaks on some uncountable subset of $S_{0}$.

Proof. Let $\Delta$ be a closed uncountable subset of the unit circle of (1-dimensional) Lebesgue measure zero. Let $\phi$ be a continuous function on $K$ analy tic at each point of int $K$ and such that $\phi^{-1}(1)=\Delta$ and $\phi^{-1}(K \backslash \Delta) C$ int $K$ (cf. $[9$, p. 81]). Let $g=\phi \circ h$, where $h \in A$ is chosen as in Proposition 1. Since $\phi$ is a uniform limit (in $K$ ) of a sequence of polynomials, say $\left(W_{n}\right)$, the function $g$ is the uniform limit of the sequence $\left(W_{n} \circ h\right)$. Hence $g$ belongs to $A$.

Clearly $g$ peaks on the set $h^{-1}(\Delta) \subset S_{0}$.

\section{The main result.}

TheOREm 1. Let $A$ be a function algebra on a compact metric space $S$. Then the following conditions are equivalent.

(a) $S$ is uncountable.

(b) $A^{*}$ is nonseparable.

(c) $B(A)$ is uncountable.

(d) There exists a subset $T$ of $S$ homeomorphic to the Cantor discontinuum and a linear operator $u: C(T) \rightarrow A$ such that for every $f \in C(T)$, $\|u f\|=\|f\|$ and $(u f)(s)=f(s)$ for $s \in T$.

(e) A contains a closed linear subspace which is isometrically isomorphic to the space of all continuous functions on the Cantor discontinuum and which is the range of a projection of norm one from $A$.

(f) Every separable normed linear space is linearly isometric to a linear subspace of $A$.

Proof. (a) $\rightarrow$ (b). If $S$ is uncountable, then $M(S)=[C(S)]^{*}$ is nonseparable (because if $s^{\prime} \neq s^{\prime \prime}$, then $\left\|\eta_{s^{\prime}}-\eta_{s^{\prime \prime}}\right\|=2$ for arbitrary $s^{\prime}$ and $s^{\prime \prime}$ in $S$ ). Therefore we can restrict our attention to the case where $A \neq C(S)$. In this case there is a maximal antisymmetric set, say $S_{1}$, for $A$ which contains more than one point (cf. [2], [6]). Let $r$ be the operator which assigns to each $f$ in $A$ its restriction to $S_{1}$ and let $r A=A_{1}$. By results of Bishop [2] (cf. also [6]) and Šilov [17], $A_{1}$ is an antisymmetric function algebra on $S_{1}$. Since $r$ maps $A$ onto $A_{1}$, the adjoint map $r^{*}$ is a linear homeomorphism from $A_{1}^{*}$ in to $A^{*}[5, \mathrm{p} .488]$. Therefore it is enough to show that $A_{1}^{*}$ is nonseparable. To prove this observe first that $B\left(A_{1}\right)$ has no isolated points. Otherwise, $A_{1}$ would contain a nonconstant idempotent which contradicts the antisymmetry of $A_{1}$. (Indeed, let $X$ be a function algebra on $T$ and let $t_{0}$ be an isolated point of $B(X)$. Then $[15$, p. 53] there is an $f$ in $X$ such that $\|f\|=f\left(t_{0}\right)=1$ and $|f(t)| \leqq \frac{3}{4}$ for $t \neq t_{0}$. Clearly $\left\|f^{p}-f^{q}\right\| \leqq\left(\frac{3}{4}\right)^{p}+\left(\frac{3}{4}\right)^{q}$ for $p, q=1,2, \cdots$. Hence $\left(f^{n}\right)$ is a Cauchy sequence. Let $e=\lim _{n} f^{n}$; then 
$e\left(t_{0}\right)=1$ and $e(t)=0$ for $t \neq t_{0}$. Thus, $e$ is a nonconstant idempotent.) Thus $B\left(A_{1}\right)$ is uncountable, because it has no isolated points and it is a $G_{\delta}$ in a compact metric space (cf. $[8, \mathrm{p} .137, \mathrm{X}]$ )

Let us set

$$
x_{s}^{*} f=f(s) \quad \text { for } f \in A_{1} \text { and } s \in B\left(A_{1}\right) .
$$

Then $x_{s}^{*} \in A_{1}^{*}$ and $\left\|x_{s}^{*}\right\| \leqq 1$ for $s \in B\left(A_{1}\right)$, and $\left\|x_{s_{1}}^{*}-x_{s_{2}}^{*}\right\|=2$ for $s_{1} \neq s_{2}$. Indeed, if $f_{i}$ peaks at $s_{i}(i=1,2)$, then

$$
\lim _{n}\left\|f_{1}^{n}-f_{2}^{n}\right\|=1 \text { and } 2 \geqq\left\|x_{s_{1}}^{*}-x_{s_{2}}^{*}\right\| \geqq \lim _{n}\left(x_{s_{1}}^{*}-x_{s_{2}}^{*}\right)\left(f_{1}^{n}-f_{2}^{n}\right)=2 .
$$

Thus $A^{*}$ is nonseparable.

non (c) $\rightarrow$ non (b). Let us set

$$
M(B(A))=\left\{\mu \in M(S): \mu(f)=\int_{B(A)} f d \mu\right\} .
$$

Since (by non (c)) $B(A)$ is countable, $M(B(A)$ ) is isometrically isomorphic to the space $l_{1}$ of all absolutely convergent series. Hence $M(B(A))$ is separable. For $\mu \in M(B(A))$, let $\mu_{A}$ denote the restriction of the linear functional $\mu$ to $A$. Then, by the Bishop-Choquet-de Leeuw set up theorem (cf. $[\mathbf{1 5}$, p. 38 and p. 53]) the correspondence $\mu \rightarrow \mu_{A}$ is a linear map from $M(B(A))$ onto $A^{*}$. Hence, $A^{*}$ is separable as a continuous image of a separable Banach space.

(c) $\rightarrow(d)$. According to a result of Bishop [3] and the Main Theorem of [12] it is enough to show

$\left(\mathrm{d}^{\prime}\right)$ there is an uncountable compact subset $T$ of $S$ such that if $\mu \in A^{\perp}=\{\nu \in M(S): \nu(f)=0$ for $f \in A\}$, then $\mu(E)=0$ for arbitrary Borel set $E \subset T$.

Since $B(A)$ is an uncountable absolute $G_{\delta}$, it contains a proper compact perfect subset $S_{0}$ (cf. [8, p. 138, XI]). Choose (using Corollary 1) $g=g_{0,0}$ in $A$ such that $\left\|g_{0,0}\right\|=1$ and $g$ peaks on an uncountable subset of $S_{0}$. Let us set $S_{0,0}=g_{0,0}^{-1}(1)$. Since $S_{0,0}$ is uncountable we can find disjoint perfect subsets of $S_{0,0}$, say $S_{1,0}^{\prime}$ and $S_{1,1}^{\prime}$ such that diam $S_{1, t}^{\prime}$ $<\frac{1}{2}$ for $i=0,1$. Thus, applying Corollary 1 , we can find functions $g_{1,0}$ and $g_{1,1}$ in $A$ which have norm one and which peak on some uncountable subsets of $S_{1,0}^{\prime}$ and $S_{1,1}^{\prime}$ respectively. We put $S_{1, i}=g_{1, i}^{-1}(1)$ for $i=0,1$. Continuing in this manner we define inductively for $j=0,1, \cdots, 2^{n}-1$ and for $n=0,1, \cdots$ uncountable closed subsets $S_{n, j}$ of $C(A)$ and functions $g_{n, j}$ in $A$ with the following properties:

(i) $\left\|g_{n, j}\right\|=1$ and $g_{n, j}$ peaks on $S_{n, j}$,

- If $T$ is a subset of a metric space $S$, then diam $T=\sup , \in T, t \in T d(s, t)$. 
(ii) $S_{n, j} \cap S_{n, k}=\varnothing$ for $k \neq j$,

(iii) $S_{n+1,2 j} \cup S_{n+1,2 j+1} \subset S_{n, j}$,

(iv) $\operatorname{diam} S_{n, j} \leqq(n+1)^{-1}$ for $n>0$.

Let us set

$$
T=\bigcap_{n=0}^{\infty} \bigcup_{j=0}^{2^{n-1}} S_{n, j}
$$

Clearly $T$ is homeomorphic to the Cantor discontinuum (as a proper zero-dimensional perfect compact set [10, Vol. II, p. 58]). We shall show that $T$ satisfies the condition $\left(\mathrm{d}^{\prime}\right)$. To do this it is enough to show that if $\mu \in A^{\perp}$, then $\mu\left(T \cap S_{m, k}\right)=0$ for $k=0,1, \cdots, 2^{m-1}$ and for $m=0,1, \cdots$, because the sets $S_{m, k}$ generate the field of all Borel subsets of $T$.

For a fixed pair $(m, k)$ with $0 \leqq k \leqq 2^{m}$ and $m=0,1, \cdots$, let us set

$$
N_{p}=\left\{j \leqq 2^{p}-1: S_{p, j} \subset S_{m, k}\right\} \quad(p=m, m+1, \cdots) .
$$

Then

$$
T \cap S_{m, k}=\bigcap_{n \geqq m} \bigcup_{j \in N_{p}} S_{p, j}
$$

Let $\epsilon>0$. Since $\mu$ is a finite regular Borel measure, there is an index $p \geqq m$ such that

$$
\mu\left(\bigcup_{j \in N_{p}} S_{p, j} \backslash T \cap S_{m, k}\right)<\epsilon .
$$

Let us set

$$
f_{n}=\sum_{j \in N_{p}} g_{p, j}^{n} \quad(n=1,2, \cdots) .
$$

Clearly, by (i), $\left\|f_{n}\right\| \leqq \sum_{j=1}^{2^{p}-1}\left\|g_{p, \lambda}^{n}\right\|=2^{p}-1$ for $n=1,2, \cdots$ and $g_{p, j}^{n}$ converges pointwise to the characteristic function of $S_{p, j}$ $\left(j=0,1, \cdots, 2^{p}-1\right)$. Hence, by (ii), the sequence $f_{n}$ converges to the characteristic function of the set $\bigcup_{j \in N_{p}} S_{p, j}$. Therefore, since $\mu \in A^{\perp}$, the Lebesgue dominated convergence theorem implies

$$
0=\lim _{n} \mu\left(f_{n}\right)=\lim _{n} \int_{S} f_{n}(t) \mu(d t)=\int_{S} \lim _{n} f_{n}(t) \mu(d t)=\mu\left(\bigcup_{j \in N_{p}} S_{p, j}\right)
$$

Thus $\left|\mu\left(T \cap S_{m, k}\right)\right|<\epsilon$, because of the choice of the index $p$. Since $\epsilon$ is an arbitrary positive number, $\mu\left(T \cap S_{m, k}\right)=0$.

$(d) \rightarrow(e)$. This follows from [12, Proposition 1].

$(e) \rightarrow(f)$. This follows from the fact that every separable normed 
linear space is linearly isometric to a linear subspace of the space of all continuous functions on the Cantor discontinuum [4, p. 93 (6)].

non (a) $\rightarrow$ non (f). If $S$ is countable, then $M(S)$ is linearly isometric to $l_{1}$, in particular $M(S)$ is separable [16], [17]. Thus every linear subspace $X$ of $C(S)$, in particular every subspace of $A$, has a separable dual, because $X^{*}$ is a continuous image (by the restriction map) of $M(S)$.

Corollary 2. Let $A$ be a function algebra on a compact metric space $S$ and suppose that there exists a (bounded) projection from $C(S)$ onto $A$. Then $A$ is linearly homeomorphic to $C(S)$.

Proof. If $S$ is countable, then by a result of Rudin [16], $A=C(S)$. If $S$ is uncountable, then by a result of Miljutin (cf. [11], [13, Theorem 8.5 ]) $C(S)$ is linearly homeomorphic to $C(\mathcal{C})$, where $\mathfrak{e}$ denotes the Cantor discontinuum. Thus by the assumption of the corollary, $A$

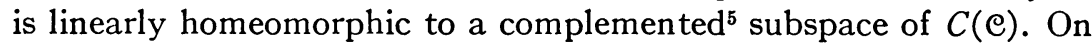
the other hand, by Theorem 1 (condition (e)) $A$ contains a complemented subspace which is linearly isometric to $C(\mathcal{e})$. Thus, by [13, Proposition 8.3], $A$ is linearly homeomorphic to $C(\mathbb{C})$ or equivalently to $C(S)$.

Let us note that Corollary 2 is related to the conjecture of Glicksberg [7], that if $A$ is a closed complemented subalgebra of $C(S), S$ compact Hausdorff, then $A$ is selfadjoint. Indeed if this conjecture is true, then the Stone-Weierstrass theorem will imply that a complemented function algebra on a compact Hausdorff space $S$ is the whole space $C(S)$.

\section{REFERENCES}

1. E. Bishop, A minimal boundary for function algebras, Pacific J. Math. 9 (1959), 624-642.

2. - A generalization of the Stone-Weierstrass theorem, Pacific J. Math. 11 (1961), 777-783.

3. - A general Rudin-Carleson theorem, Proc. Amer. Math. Soc. 13 (1962), 140-143.

4. M. M. Day, Normed linear spaces, Academic Press, New York, 1962.

5. N. Dunford and J. Schwartz, Linear operators, Part I, Interscience, New York, 1958.

6. I. Glicksberg, Measures orthogonal to algebras and sets of antisymmetry, Trans. Amer. Math. Soc. 105 (1962), 415-435.

7. - Some uncomplemented function algebras, Trans. Amer. Math. Soc. 111 (1964), 121-137.

8. F. Hausdorff, Mengenlehre, Dover, New York, 1944.

- A linear subspace $Y$ of a Banach space $X$ is said to be complemented in $X$ if there is a (bounded linear) projection from $X$ onto $Y$. 
9. K. Hoffman, Banach spaces of analytic functions, Prentice-Hall, Englewood Cliffs, N. J., 1962.

10. K. Kuratowski, Topologie II, Monogr. Mat., Warsaw, 1961.

11. A. A. Miljutin, Isomorphism of spaces of continuous functions on compacts of the power continuum, Teor. Funk. Funkcional. Anal. i Priložen. (Function Theory, Functional Analysis and their Applications) 2 (1966), 150-156. (Russian)

12. A. Pełczyński, On simultaneous extension of continuous functions, Studia Math. 24 (1964), 285-304.

13. - Linear extensions, linear averagings and their application to linear topological classification of spaces of continuous functions, Rozprawy Mat. (to appear).

14. A. Pelczyński and Z. Semadeni, Spaces of continuous functions (III), Studia Math. 18 (1959), 211-222.

15. R. R. Phelps, Lectures on Choquet's Theorem, Van Nostrand, Princeton, N.J., 1965.

16. W. Rudin, Continuous functions on compact spaces without perfect subsets, Proc. Amer. Math. Soc. 8 (1957), 39-42.

17. G. E. Silov, On rings of functions with uniform convergence, Ukrain. Mat. $\check{Z}$. 3 (1951), 404-411. (Russian)

UNIVERSITY OF WARSAW AND

UNIVERSITY OF WASHINGTON 